predissociation limit of $C,{ }^{2} \Sigma$, all remaining states $A, B, D$ and $E$ dissociating into ${ }^{3} P$. This correlation of mine was suggested as an alternative in the paper of More and Cornell.

It is convenient to undertake the correlation $\mathrm{SrH}-\mathrm{Sr}+\mathrm{H}$ in connexion with the similar process $\mathrm{CaH}-\mathrm{Ca}+\mathrm{H}$, because the energy schemes in both cases are almost identical ${ }^{2}$. Further, it must be regarded as most probable that the ground-level $(N)$ of the hydrides dissociates into ${ }^{1} S$ and the $A$ and $B$ levels go to ${ }^{3} P$. Accordingly, if the ${ }^{3} P$ or ${ }^{3} D$ levels of the atoms are taken as predissociation limit, two different dissociation values are obtained for the $N, A$ and $B$ states. A comparison between these two sets of values and those which are obtained with the Rydberg extrapolation method, shows that the choice of ${ }^{3} P$ as predissociation limit is much more probable than that of ${ }^{3} \mathrm{D}$. The same result has also been obtained by Humphreys and Fredrickson ${ }^{3}$, although the dissociation values given by them are not correct. It is easy to see that the BirgeSponer extrapolation in these cases gives by no means correct dissociation values, because the Birge-Sponer values are about $5,000 \mathrm{~cm} .^{-1}$ higher than the highest possible dissociation values in the $N, A$ and $B$ states of $\mathrm{CaH}$ and $\mathrm{SrH}$.

A fuller account of these problems will appear in the Zeitschrift für Physik.

Department of Physies,

\section{Birger Grundström.}

University of Stockholm. July 30.

'More, K. R., and Cornell, S. D., Phys. Rev, 53, 806 (1938).

? Grundström, B., Z. Phys., 99, 595 (1936); Dissertation, Stockholm, 1936. Funke, G. W., and Grundström, B., Z. Phys., 100, 293 (1936). ${ }^{3}$ Humphreys, R. F., and Fredrickson, W. R., Phys. Rev., 50, 542 (1936).

\section{Kramers Law : A Correction}

IN a recent letter ${ }^{1}$ it was shown that the number of arrivals, $p_{n}$, in the $n^{\text {th }}$ quantum state of the hydrogen atom in a discharge tube and in the planetary nebulæ was not the same as the number of captures predicted by Kramers' Law. The arrivals, $p_{n}$, were computed from measured line intensities for $n \supseteq 3$, and the number of captures on $n=2$ was obtained directly from the Balmer continuous emission, $B a_{c}$. If, as convincing arguments ${ }^{2}$ appeared to show, these observed arrivals, $p_{n}$, were almost wholly due to captures of free electrons, it followed that Kramers' Law was wrong. This view was strengthened in the case of the discharge tube by a measurement of the Paschen continuous emission, $P a_{c}$, which gave directly the number of captures on $n=3$, and which was thought to be of the same magnitude as $p_{3}$.

The infra-red measurements of the $P a_{c}$ have recently been repeated with greater accuracy and a numerical error found in the earlier measure. It now appears that the ratio of $P a_{c}$ to $B a_{c}$ is very closely that predicted by Kramers' Law, namely, $0 \cdot 06^{3}$; therefore the law is correct for hydrogen, as expected theoretically. It is evident, then, that $p_{3}$ is one thousand times greater than the number of captures on $n=3$ both in the electrodeless ring discharge and in the nebulæ; in other words, excitations to the third quantum level are about one thousand times as frequent as captures of free electrons there. This fact will necessitate a revision of the prevailing theory of nebular emission.
Thus, though Kramers' Law should not be used in calculating the absorption of the alkalis and the nonhydrogenic elements in a stellar atmosphere, these revised and extended measurements indicate the validity of the law for atomic hydrogen.

University Observatory,

T. L. Page.

Oxford.

Aug. 31.

${ }^{1}$ Nature, 141, 1137 (June 25, 1938).

2 Zanstra, H., Astrophys. J., 65, 50 (1927); Plaskett, H. H., Pub. Dom. Astro. Obs., 4, 187'(1928).

${ }^{3}$ Note added in proof: In a letter in NATURE, 142, 433 (September $3,1938)$, Menzel has directed attention to the significance of this test, and has made the forecast, now verified, that it would conflrm the validity of Kramers' Law.

\section{The Rotating Pendulum and the State of Adaptation of the Eye}

The experiments of Lythgoe ${ }^{1}$ on the rotating pendulum show that the latent period of the eye is not directly related to the brightness perception of a viewed object. The latent period seems to be more closely related to the sensitivity of the eye : with higher sensitivity it is mostly longer, with lower sensitivity shorter. Lythgoe was led to assume that nervous interaction tends to shorten the latent period. Crawford ${ }^{2}$ states that the effect is almost entirely in accord with the hypothesis that the latent period varies in the same sense as the concentration of photochemical substance in the retina, and concludes that the effect might therefore provide a simple measure of the concentration of photochemical substance in the retina.

Experiments on the change of sensitivity of the fovea under influence of a glaring light source placed somewhere else in the field of view point strongly against Crawford's and in favour of Lythgoe's hypothesis.

It can be shown by means of a binocular method ${ }^{3,4}$ that the presence of a glare source in the field of view causes the sensitivity of the fovea to drop to a much lower level within $0 \cdot 1$ second ( $\alpha$-adaptation). If the sensitivity were determined solely by the concentration of a photochemical substance, $\alpha$ adaptation ought to be due either to the stray light within the eye or to a diffusion of photochemical substances over the retina. It can be proved, by measurements on diascleral re-illumination, that $\alpha$-adaptation is not due to this stray light, but to an influence originating in the strongly illuminated part of the retina. By measurements on the velocity of $\alpha$-adaptation over the retina, a value was obtained which is far too great to be accounted for by processes of diffusion.

$\alpha$-Adaptation might be of a purely electrical nature, due to the leakage currents which are known to be produced within the eye when a point of the retina is illuminated. Although this hypothesis qualitatively accounts for many of the observed characteristics of $\alpha$-adaptation, it cannot account for all of them, especially those with coloured light. After excluding the possible explanations of purely physical or chemical nature, we are thus left with only one possibility to account for $\alpha$-adaptation : an inhibitive influence transmitted by the retinal synapses from the illuminated area of the retina towards the fovea.

Against Crawford's assumption also is the fact that for the one form of adaptation for which photochemical changes seem definitely responsible, namely, 